\title{
One Health approaches require community engagement, education, and international collaborations-a lesson from Rwanda
}

\author{
One Health approaches recognize the links between the environment, animals, and human disease, but \\ these approaches are successful only with bottom-up community engagement, education, and international \\ collaborations.
}

\section{Phaedra Henley, Gloria Igihozo and Laura Wotton}

( ne Health is a new concept for many, yet it is a concept that has, historically and currently, impacted lives in every population globally. Recognizing the interconnection among people, animals and their shared environment, One Health principles have become especially relevant to how the COVID-19 pandemic started, presumably with a virus jumping from its bat host to an undetermined species and then to humans, with devastating effect.

For the first time in the lifetime of many people, every country has a shared health threat, yet there has been a wide variance in responses, depending on the location. Countries whose governance systems have prioritized formal coordination across multiple sectors informed by the One Health approach have, as a general rule, performed better in curbing SARS-CoV-2, a virus that crosses borders. Using an institutionalized One Health approach, both the East Africa Community and West Africa have leveraged regional partnerships in order to collaborate to fight COVID-19 ${ }^{1,2}$. Particular success stories include Burkina Faso, Liberia and Rwanda, low- to middle-income countries that have, with scant resources and budget, fared better against COVID-19 than did many of their global north neighbors ${ }^{3}$.

\section{Community engagement}

In sub-Saharan Africa, zoonotic infections account for $26 \%$ of the years of healthy life lost to infectious diseases, and this figure is only set to grow as increasingly dense populations collide with the animal world and ecosystem services deteriorate as a result of forests being turned into land for farming and grazing ${ }^{4}$. Consequently, many African countries have developed evidence-based frameworks within their governance structure to build resilience to zoonoses and other environmental health threats such as climate change ${ }^{5}$. In Rwanda, this has manifested itself in

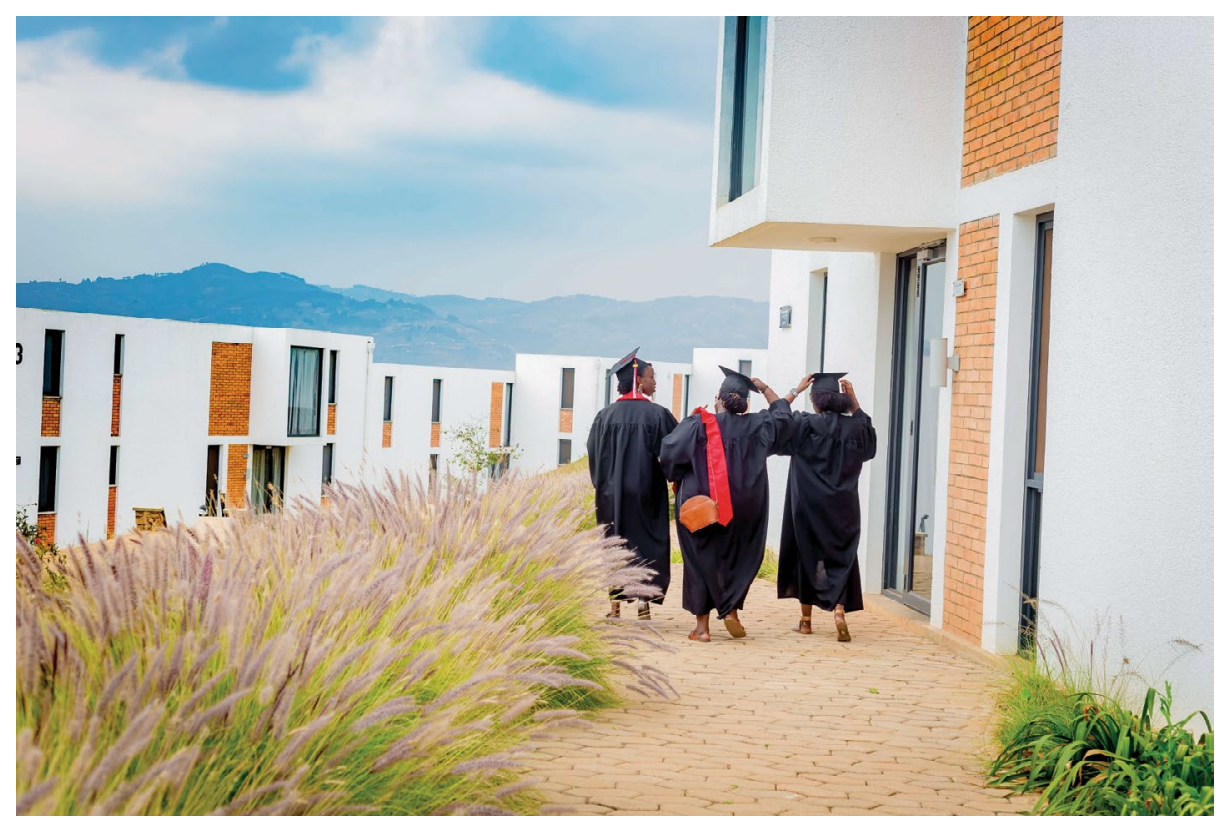

Students attending The University of Global Health Equity in Rwanda's Northern province. Credit: 64 Waves for UGHE

infrastructure, including a One Health Steering Committee ${ }^{6,7}$, and the ongoing development of a One Health workforce aimed at mobilizing experts across disciplines to prepare for, coordinate responses to and manage infectious-disease outbreaks. Rwanda's neighbor Uganda has also formalized One Health approaches through its National One Health Platform, a roadmap that translates its long history of zoonotic diseases into a strategic approach to multi-sectoral preparedness and response that involves many sectors, including health, agriculture, wildlife, and water and the environment ${ }^{8}$.

Although awareness of One Health as an approach is steadily growing, unless this is translated into action, the world remains vulnerable to future outbreaks ${ }^{9}$. Practical frameworks are needed to design and implement One Health programs, policies, legislation and research that, although initiated and adopted by governments, must engage communities. Community members, particularly those living in rural settings, are among those most vulnerable to changes in environmental and animal health ${ }^{10}$. Community members are therefore essential to informing One Health policy, and their networks are vital in putting One Health approaches into practice.

Governments need to mobilize community representatives as part of their risk-reduction strategy; these representatives are essential sentinels of surveillance for monitoring and communicating changes in animal, human and environmental health. For 
many communities, sentinels with local expertise and experience are not new, and they are able to recognize and act on One Health threats. Rwanda has leveraged its decentralized network of community health workers, community-based animal-health workers, healthcare facilities, park rangers, border agents, farmers, and domestic-animal owners as sentinels for monitoring potential zoonotic disease outbreaks ${ }^{11}$.

\section{Education}

Education is central to the integration of One Health approaches with national strategies. Educational institutions should support government and community efforts by initiating formal frameworks to teach One Health principles so that they can be easily applied in a practical setting.

The University of Global Health Equity (https://www.ughe.org), a global health institution in Rwanda, is championing One Health approaches through a pioneering educational model. Through the university's Center for One Health, students are trained to think holistically, prioritizing collaborative, multi-sectoral partnerships, and are equipped with the tools to design and deliver innovative, equitable and evidence-based solutions to complex global challenges such as COVID-19. The unique positioning of this university in Rwanda's remote Northern province provides a stimulating environment for students to see One Health approaches in action by learning directly from farmers, community members and livestock owners and the protective measures that these practitioners employ against zoonotic disease transmission.

\section{International collaborations}

Transnational collaborations are vital for the uptake and implementation of One Health approaches. Recognizing this need, the University of Global Health Equity, its Peruvian sister organization Socios En Salud (https://sociosensalud.org.pe) and their US-based parent organization Partners in Health (https://www.pih.org) have developed a virtual short course, 'Pandemic Preparedness and Response and Dynamic Health System Resilience ${ }^{12}$, that includes case studies from different countries, to encourage transnational coordination, collaboration and communication. Course participants learn the fundamental principles of equity-centered and evidence-based pandemic preparedness and response, the role of leadership, and the role of policymakers and frontline health workers in systems readiness, and participants leave equipped with the tools and resources to provide an effective response to complex domestic and global health threats.

One Health approaches, when adopted by governments and effectively operationalized at the community level, are vital response, recovery and resilience mechanisms that have already led to considerable health gains in many low- and middle-income countries ${ }^{3,13}$. So that these can have maximum impact, and for lessons to be learned from the COVID-19 pandemic, investment must be made in education that not only builds awareness of One Health principles but also provides practical frameworks with which to implement these principles at local, national and international levels.

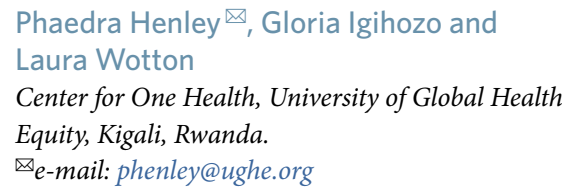

Published online: 20 May 2021

https://doi.org/10.1038/s41591-021-01350-5

\section{References}

1. East African Community. https://www.eac.int/press-releases/ 147-health/1916-eac-secretariat-contributes-to-establishinga-one-health-workforce-in-the-region (15 December 2020).

2. Vernon, P. European Think Tank Group https://ettg. eu/2021/01/25/lessons-from-covid-19-for-a-stronger-onehealth-partnership-between-the-eu-and-west-africa/ (25 January 2021)

3. Binagwaho, A. \& Mathewos, K. MedicalNewsToday https://www.medicalnewstoday.com/articles/ what-explains-africas-successful-response-to-the-covid19-pandemic (2020)

4. Grace, D., Gilbert, J., Randolph, T. \& Kang'ethe, E. Trop. Anim. Health Prod. 44, 67-73 (2012).

5. Nhamo, G. \& Muchuru, S. Jamba 11, 644 (2019).

6. Rwanda One Health Steering Committee. One Health Strategic Plan (2014-2018) 1-74 (2014).

7. Rwanda One Health Steering Committee. One Health Policy 1-33 (2019a)

8. Buregyeya, E. et al. J. Epidemiol. Glob. Health 10, 250-257 (2020).

9. Destoumieux-Garzón, D. et al. Front. Vet. Sci. 5, 14 (2018).

10. Asian Development Bank. https://www.adb.org//sites/default/ files/publication/42089/climate-change-rural-communities-gms. pdf (2014).

11. Nyatanyi, T. et al. BMJ Glob Health 2, e000121 (2017).

12. University of Global Health Equity. https://ughe.org/ppr/ (2021).

13. Cleaveland, S. et al. Phil Trans R Soc B. 372, 20160168 (2017).

Author contributions

P.H. conceived of the work and interpreted the data; G.I. collected and interpreted the data; L.W. wrote the Comment; and all authors approved the final version.

Competing interests

The authors declare no competing interests. 